\title{
Postmenopausal complete labial fusion: a rare case study
}

\author{
Prajakta Shirish Tumbal $^{1 *}$, Rabin Majumdar ${ }^{2}$
}

\author{
${ }^{1}$ Department of Obstetrics and Gynaecology, ARMCH, Solapur, Maharashtra, India \\ ${ }^{2}$ Department of Obstetrics and Gynaecology, KEM, Pune, Maharashtra, India
}

Received: 24 February 2016

Revised: 21 March 2016

Accepted: 30 March 2016

\author{
*Correspondence: \\ Dr. Prajakta Shirish Tumbal, \\ E-mail: prajshirish_21@yahoo.com
}

Copyright: (c) the author(s), publisher and licensee Medip Academy. This is an open-access article distributed under the terms of the Creative Commons Attribution Non-Commercial License, which permits unrestricted non-commercial

\begin{abstract}
Complete labial fusion is a rare clinical entity in postmenopausal women. It occurs when the labia minora have become fused in midline through either flimsy or dense adhesions, forming a raphe. Labial fusion has been associated with local irritation and inflammation in the setting of a hypoestrogenic state. Topical treatment is usually recommended prior to manual separation. However, some postmenopausal women have been refractory to conservative management, and surgery may then be required. Here we present a case of a 62 yrs old postmenopausal woman, parity three; live three with previous 3 caesarean sections, operated case of Abdominal Panhysterectomy 11 yrs back for fibroid uterus was admitted to private hospital in April 2013 with complaints of Incomplete evacuation of urine, Passing urine on standing after squatting position, Urgency of urination and dribbling of urine for some time after urination since last one year. General examination and investigations were done which were normal. Patient required surgical excision of fused labial fold to relieve the symptoms.
\end{abstract}

Keywords: Labial fusion, Panhysterectomy, Postmenopausal, Urethroplasty

\section{INTRODUCTION}

Labial adhesions mean that the labia minora are fused in midline through either flimsy or dense adhesions. Labial fusion or adhesions usually occurs in young girls and at any age prior to puberty and are known as primary labial adhesions. Secondary labial adhesions usually occur due to estrogen deficiency, particularly in non-sexually active women after menopause. Fusion may be partial or complete. Fusion usually starts at the posterior fourchette and progresses towards the clitoris. Labial adhesions are usually caused by scarring or fibrosis that forms after severe inflammatory skin disease, surgery or trauma. Most common causes are oestrogen deficiency associated with postmenopausal atrophic vaginitis. Considering the relative frequency of post-menopausal urethritis and vaginitis, complete labial fusion as a result of this condition alone is poorly recorded. In contrast to the paediatric population in which the fusion usually involves the labia minora, in postmenopausal women adhesion may also involve the labia majora. ${ }^{1}$ Severe labial fusion can sometimes leave only a small pinhole through which urine can escape, and the vagina fills with urine causing urocolpos and dribbling. These findings can predispose patient to ascending infections.

\section{CASE REPORT}

A 62 yrs old postmenopausal woman, parity three live three with previous 3 caesarean sections, operated case of Abdominal Panhysterectomy 11 yrs back for fibroid uterus was admitted to private hospital in April 2013 with complaints of Incomplete evacuation of urine, Passing urine on standing after squatting position, Urgency of urination and dribbling of urine for some time after urination since last one year. She denied history of something coming out of vagina, Stress Urinary Incontinence or burning micturition, per vaginal white discharge or per vaginal bleeding. Patient was operated for Abdominal Panhysterectomy 11 yrs back for fibroid 
uterus. She was postmenopausal since last 20 years and was not sexually active since last 15 years. Her general physical examination was normal with stable vitals. Past medical history was insignificant. Per abdominal examination was unremarkable. On per vaginal examination, there was evidence of complete fusion of labia minora from clitoris to the posterior forchette with tiny hole at anterior $1 / 3^{\text {rd }}$ of introitus through which she was voiding. On applying pressure over the site of fusion urine leak was seen from that hole. Urethral orifice was not visible and there was no evidence of clitoromegaly. On per rectal examination a patent vaginal canal was palpated. Pubic hair was present but sparse and appeared to be consistent with her post-menopausal status .External genitalia were free of typical cutaneous signs of lichen sclerosis or lichen planus. A provisional diagnosis of complete labial fusion was made. Investigation showed normal renal profile. Ultrasonography (USG) of abdomen and pelvis was unremarkable but post void residual was significant. Computed Tomography (CT) of abdomen and pelvis was also within normal limits.

\section{Management}

In symptomatic patients there are typically four methods for treatment including:

\section{Oral estrogen}

2. Topical estrogen/steroids

3. Manual separation without anaesthesia

4. Blunt or sharp separation with local, regional or general anaesthesia.

Patient was posted for excision of labial fusion under spinal anaesthesia. Complete labial excision was done to prevent refusion. Surgical correction was relatively simple. Midline fusion was divided and opened into vagina. Vagina showed features of soreness due to stagnation of urine for long time. The urethra was mildly narrowed. Skin edges were sutured to the vaginal epithelium. The patient recovered well post-operatively and was discharged home after two days. She was advised local application of estrogen cream into the vagina.

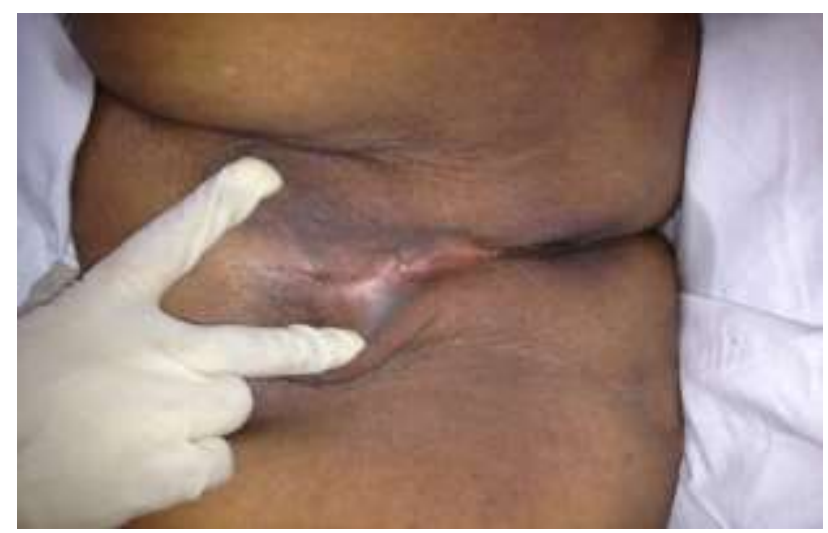

There was no evidence of adhesions inside vagina. Lower $1 / 3^{\text {rd }}$ urothroplasty was done as there was involvement of lower urethra. Patient was catheterised and catheter was removed after $48 \mathrm{hrs}$. Postoperatively patient was able to pass urine without any complaints. On follow up examination the patient has been able to maintain an open introitus and normal urine flow with continued use of vaginal estrogen cream and simultaneous vaginal dilatation for 20 minutes 3 nights per week.

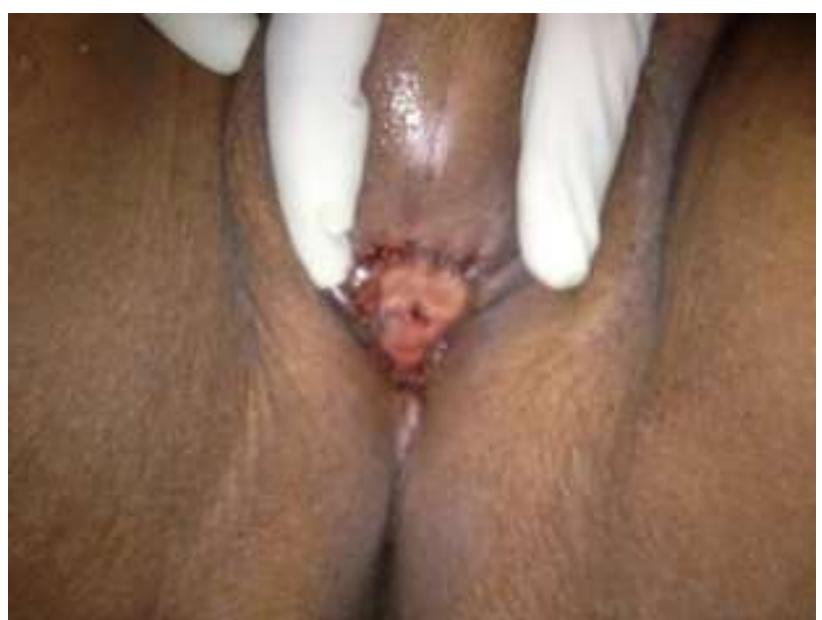

Figure 2: Post-operative.

\section{DISCUSSION}

Labial fusion or adhesion typically seen in young girls and at any age prior to puberty is usually asymptomatic and may be managed expectantly or with topical estrogen, often resolving after menarche. ${ }^{2}$ However, postmenopausal women can experience significant symptoms including urocolpos, inability to void, infections, and interference with sexual activity .Fusion may be partial or complete. Fusion usually starts at posterior fourchette and progresses towards the clitoris. Labial adhesions are usually the result of local inflammation and chronic irritation due to hypoestrogenised vulval tissue at this age. Considering the relative frequency of post-menopausal urethritis and vaginitis, complete labial fusion as a result of this condition alone is poorly recorded. ${ }^{3}$ Dong-Ming Kuo has reported labial fusion in a 30 years old woman. ${ }^{4} \mathrm{We}$ could assume that labial fusion in our patient is due to the effect of vulval hypoestrogenisation. It is recommended that topical treatment be attempted prior to manual separation, however some postmenopausal women have been refractory to conservative management, and surgery may be required. In long standing cases of labial fusion it is also important to remember to perform a complete assessment of reproductive organs as labial fusion may limit evaluation or mask common findings that lead to to diagnosis of malignancy such as vaginal bleeding or a palpable mass.

Figure 1: Pre-operative. 


\section{CONCLUSION}

Postmenopausal labial fusion is a very rare condition. Very few cases have been documented in literature till now. This condition should be properly evaluated and it can be managed with simple surgical procedure.

Funding: No funding sources Conflict of interest: None declared

Ethical approval: Not required

\section{REFERENCES}

1. Chuong CJ, Hodgkinson P. Labial adhesion presenting as Urinary incontinence in postmenopausal women .Obstetrics and Gynaecology. 1984;64:81s-4s.

2. Kumetz LM, Quint EH, Fisseha S, Smith YR. Estrogen treatment success in Recurrent and Persistent Labial Agglutination. J Pediatr Adolesc Gynecol. 2006;19(6):381-4.

3. Nicole CS, Dwyer PL. Labial fusion causing voiding difficulty and urinary incontinence. Australian and New Zealand Journal of Obstetrics and Gynaecology. 1999:39(3):391-3.

4. Kuo DM, Chuang CK, Hsieh CC. Labial fusion in thirty year old woman. Acta Obstet Gynecol Scand. 1998:77:697-8.

Cite this article as: Tumbal PS, Majumdar R.

Postmenopausal complete labial fusion: a rare case study. Int J Reprod Contracept Obstet Gynecol 2016;5:1646-8. 